\title{
Phenotypic characterisation and drug sensitivity testing of mycobacteria isolated from extra-pulmonary tuberculosis
}

\author{
Gurung R ${ }^{1}$, Bhattacharya SK ${ }^{1}$, Pradhan $\mathbf{B}^{1}$, Gurung $\mathrm{S}^{1}$, Singh $\mathbf{Y I}^{2}$ \\ ${ }^{1}$ B. P. Koirala Institute of Health Sciences, Dharan, Nepal. ${ }^{2}$ Manipal College of Medical Sciences, Pokhara, Nepal
}

\begin{abstract}
Background: Tuberculosis (TB) is a major cause of morbidity and mortality globally, with most cases occurring in developing countries. The importance of extra pulmonary tuberculosis (EPTB) among all forms of tuberculosis has not yet been ascertained in developing countries

Objective: To identify and perform phenotypic characterization of clinically important mycobacterium isolates from extra-pulmonary sites along with drug susceptibility testing.

Materials and methods: A total of 513 specimens from patients of (EPTB) with varied presentation were studied. Speciation and characterization of isolates were done on the basis of growth and biochemical characteristics. Drug susceptibility testing for mycobacterium isolates was done by proportion method.

Results: Fifty four patients clinically suspected to have extra-pulmonary tuberculosis were culture positive for mycobacteria. On characterization 48 of the 54 isolates were identified as M. tuberculosis, 4 as M. bovis and 2 were M. avium/ intracelulare.

Drug susceptibility testing was done for the both M. tuberculosis as well as M. bovis. In single drug resistance, Streptomycin was highest followed by Isoniazid, Ethambutol and Refampicin. Multi drug resistance (MDR) was found in 6 isolates and it was observed only in group I cases.

Conclusion: EPTB accounts for 10-15 percent of all cases of tuberculosis. Mycobacterium was present in 10.5 percent samples. 48 isolates out of 54 samples were found to be M. tuberculosis. The maximum numbers of M. tuberculosis were isolated from lymph node aspiration.
\end{abstract}

Key words: Phenotypic characterization, drug susceptibility, extra pulmonary tuberculosis

$\mathrm{T}$ uberculosis (TB) is a major cause of morbidity and mortality globally, with most cases occurring in developing countries ${ }^{1}$. In Nepal, about 45 percent of the total population is infected with TB, out of which 60 percent are in the adult group. Every year 40,000 people develop active TB, of whom 20,000 have infectious pulmonary disease, and $5000-7000$ people continue to die every year from this disease ${ }^{2}$.

TB is a disease that can affect many systems in the body $^{3}$. The importance of EPTB among all forms of tuberculosis has not yet been ascertained in developing countries. This is due to the difficulty of the clinical and laboratory diagnosis by culture confirmation ${ }^{4}$.

The prevalence of non-tuberculous mycobacteria (NTM) both in the environment and as pathogen varies in different parts of the world ${ }^{5}$. Identification of NTM is important for epidemiological studies and a better understanding of the pathogenesis of the disease, they cause $^{6}$.
The diagnosis of EPTB poses a special challenge, as it is often missed or misdiagnosed due to its atypical presentations. The first step in its diagnosis is awareness and a high index of suspicion by the physician ${ }^{7}$. Even then, it is difficult to isolate $M$. tuberculosis due to the small number of organisms present at these sites ${ }^{4}$. Various international studies in the world have focused on the problem of EPTB, reporting a high frequency ${ }^{4,7}$. However, this aspect of tuberculosis has not been properly investigated in Nepal. Therefore, this study was undertaken to identify and perform phenotypic characterisation of clinically important Mycobacterium isolates from extra-pulmonary sites along with drug susceptibility testing.

Correspondence

Dr. Rajendra Gurung

Associate Professor

Department of Microbiology

BPKIHS, Dharan

E-mail: drrajendragrg@gmail.com 
Materials and methods

This was a laboratory based descriptive study conducted from $1^{\text {st }}$ January 2004 - 31 $1^{\text {st }}$ December 2004, at the tuberculosis laboratory of the Department of Microbiology, B.P. Koirala Institute of Health and Sciences, Dharan, Nepal.

All the specimen received from extra-pulmonary sites were included in this study. Specimens were collected and transported in clean, leak proof and labeled containers to the laboratory. These were processed by MODIFIED PETROFF method for decontamination except sterile samples under aseptic precaution on the same day for both direct microscopy and culture ${ }^{8}$.

Direct microscopy: Smears were stained by the both Auramine - Phenol stain and Ziehl Neelsen (ZN) method and reported according to NTC guidelines ${ }^{8,9}$.

Culture Isolation: Deposit was inoculated with a sterile pasture pipette over the surface of slopes of Lowenstein-Jensen (LJ) media in duplicate. The cultures were examined after $48-72$ hours to detect any contaminants. Thereafter, cultures were identified on the basis of colony morphology, pigment production and biochemical tests including niacin test, nitrate reduction test, catalase test, urase test, tellurite reduction test after 6-8 weeks of incubation or after visible growth ${ }^{8}$.

Drug susceptibility testing was done by proportion method as a standard protocol. Each strain was tested against four first line anti-tubercular drugs with control $\left(\mathrm{H} 37 \mathrm{R}_{\mathrm{v}}\right.$ ) at the following concentrations: Isoniazid 0.25 $\mu \mathrm{g} / \mathrm{ml}$, Rifampicin $40 \mu \mathrm{g} / \mathrm{ml}$, Ethambutol $2 \mu \mathrm{g} / \mathrm{ml}$ and Streptomycin $4 \mu \mathrm{g} / \mathrm{ml}^{10}$.

Statistical analysis was done in Microsoft excel programme and analyzed by SPSS 11.0 software.

\section{Results}

A total of 513 specimens from extra-pulmonary sites were included in the study. Among these 39.8 percent were from the age group 31-60 years, followed by 35.4 percent from the 15-30 years age group, 14.4 percent specimens were from paediatric age group and the rest
10.4 percent were from the elderly (i.e. $>60$ years) age group. The differences between the age groups were not significant $(\mathrm{p}<0.05)$. The male to female ratio was 1.12: 1 .

Numbers of culture positive were 54 of $513(10.5 \%)$. The age range for culture positive cases was 1 to 73 years with a median age of 29 years ( $\mathrm{SD} \pm 15.5 \mathrm{yrs}$ ). The incidence of culture positive specimens was found to be slightly higher in the male group with 62.9 percent whereas 37.1 percent were females, with a ratio of 1.7 : 1. (table 1)

Out of the 513 specimens collected, two direct smears were prepared from each specimen. One was stained using Fluorescence method (FM) and the other ZiehlNeelsen method. Culture results were used as the gold standard for assessment. The results of all the three methods are given in table 2. The differences between the tests were highly significant $(p<0.001)$.

Among the specimens which were incubated at 3 different temperatures, growth occured at $37^{\circ} \mathrm{C}$ for 54 specimens and at $25^{\circ} \mathrm{C}$ for 2 only. No growth was observed at $45^{\circ}$ C. All specimens had rough colony morphology and pigmentation test was non-photochromogenic in all specimens. On characterization, 48 out of 54 specimens were identified as M. tuberculosis, and the rest as $\mathrm{M}$. bovis (4) and M. avium / intracellulare (2).

Drug susceptibility testing was done for the both M. tuberculosis as well as M. bovis. Majority of the isolates $(94.5 \%)$ were from with no prior treatment (GroupI), and the remainder had complete treatment (Group II). Group- I was deemed to have been infected with bacilli of initial resistance and group - II of bacilli with acquired resistance.

In our study, one patient from Group II showed susceptibility to all first line anti-tubercular drugs, in vitro. Overall 32 of the 52 isolates showed resistance to one or more drugs. 30 of 32 resistant isolates were from Group I and the remaining two belonged to Group II. (table 4)

Table 1: Specimen wise Age and Sex distribution

\begin{tabular}{|l|c|c|c|}
\hline \multirow{2}{*}{ Age(Yrs) } & \multicolumn{3}{|c|}{ No. of specimens } \\
\cline { 2 - 4 } & Male (\%) & Female (\%) & Total (\%) \\
\hline$<\mathbf{1 5}$ & $41(55.4)$ & $33(44.6)$ & $74(100)$ \\
\hline $\mathbf{1 5 - 3 0}$ & $91(50)$ & $91(50)$ & $182(100)$ \\
\hline $\mathbf{3 1 - 6 0}$ & $105(51.4)$ & $99(48.6)$ & $204(100)$ \\
\hline$>\mathbf{6 0}$ & $35(66)$ & $18(34)$ & $53(100)$ \\
\hline Total & $272(53)$ & $241(47)$ & $513(100)$ \\
\hline
\end{tabular}

$(\mathrm{p}$ value $=0.200) \mathrm{SD} \pm 18.4$ 
Table 2: Results with LJ culture, ZN stain and Fluorescence stain ( $\mathrm{n}=513$ )

\begin{tabular}{|l|c|c|c|}
\hline Results & Z-N stain & Fluorescence stain & Culture positive \\
\hline Positive & $19(3.7 \%)$ & $28(5.5 \%)$ & $54(10.5 \%)$ \\
\hline Negative & $494(96.3 \%)$ & $485(94.5 \%)$ & $459(89.5 \%)$ \\
\hline
\end{tabular}

$(\mathrm{p}$ value $<0.0001)$

Table 3: Distribution of specimen positive for extra-pulmonary tuberculosis

\begin{tabular}{|l|c|c|c|c|}
\hline \multirow{2}{*}{ Specimen } & \multicolumn{4}{|c|}{ SPECIES ISOLATED } \\
\cline { 2 - 5 } & M. tuberculosis & M.bovis & M.avium/ intracellulare & Total \\
\hline Lymph node aspirate & 14 & - & - & 14 \\
\hline Urine & 9 & - & 1 & 10 \\
\hline Pleural fluid & 6 & 2 & - & 8 \\
\hline Pus from abscess & 6 & 2 & - & 8 \\
\hline Fluid aspirate (Peritonial \& ascitic) & 4 & - & 1 & 5 \\
\hline Endometrial biopsy & 3 & - & - & 3 \\
\hline CSF & 3 & - & - & 3 \\
\hline Sequestrum & 2 & - & - & 2 \\
\hline Skin tissue & 1 & - & - & 1 \\
\hline Total & 48 & 4 & 2 & 54 \\
\hline
\end{tabular}

Table 4: Initial and acquired drug resistance patterns for individual drugs

\begin{tabular}{|c|c|c|c|c|c|c|c|c|c|c|c|c|c|c|}
\hline \multirow{3}{*}{ 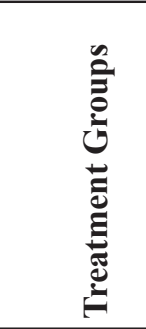 } & \multirow{3}{*}{$\begin{array}{l}\frac{n}{0} \\
. \frac{0}{0} \\
\overline{0} \\
\frac{0}{0} \\
0 \\
z\end{array}$} & \multicolumn{12}{|c|}{ Resistant } & \multirow{3}{*}{ 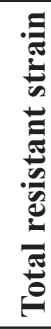 } \\
\hline & & \multicolumn{4}{|c|}{$\stackrel{00}{\Xi}$} & \multicolumn{5}{|c|}{ 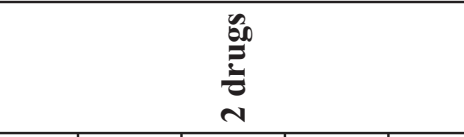 } & \multicolumn{2}{|c|}{ 党 } & \multirow{2}{*}{ 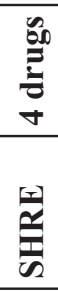 } & \\
\hline & & $\infty$ & \pm & $\simeq$ & 되 & $\underline{\underline{\Xi}}$ & $\underline{\Xi}$ & 国 & 貼 & 岳 & 氠 & $\begin{array}{l}\sqrt{2} \\
\simeq\end{array}$ & & \\
\hline Group I & 49 & 5 & 3 & 1 & 3 & 1 & 4 & 1 & 2 & 2 & 2 & 1 & 5 & 30 \\
\hline Group II & 3 & & & & & & & 1 & & & 1 & & & 2 \\
\hline Total & 52 & 5 & 3 & 1 & 3 & 1 & 4 & 2 & 2 & 2 & 3 & 1 & 5 & 32 \\
\hline
\end{tabular}

H, Isoniazid; R, Rifampicin; S, Streptomycin; E, Ethambutol Group-I, Initial drug resistance

Group-II, Acquired drug resistance

\section{Discussion}

Tuberculosis is a major cause of morbidity in Nepal. Despite the implementation of a much improved NTP, 5000 to 7000 people still die from tuberculosis each year $^{2}$. A number of studies have been published on pulmonary tuberculosis. The exact magnitude of the problem of EPTB is not known.

The diagnostic techniques for EPTB include culture, biopsy, ascertaining AFB organisms in samples, biochemical, serological and molecular methods amongst others. The Culture of $M$. tuberculosis by LJ medium is the most widely used method for determination of characteristic features of colonial morphology, growth rate and pigment production. Though the culture technique is simple, 6-8 weeks of incubation may be necessary for detection of organisms. Microscopic examination of thin layer culture plate may lead to detection of micro colonies of M. tuberculosis as early as 7 days. However, recovery of $M$. tuberculosis from this is less efficient and labor intensive ${ }^{11}$.

The present study found a culture positive rate of 10.5 percent in specimens from a wide range of sites in 513 patients clinically suspected to have EPTB which correlates with earlier findings ${ }^{12,13}$.

To the best of our knowledge, this is the first study to assess the status of EPTB, with reference to identification up to species level, and their drug susceptibility testing in Nepal. The frequency reported here is a significant 
number, because the prevalence of tuberculosis (45\%) in Nepal is high ${ }^{14}$. The real figure of EPTB might be even higher.

In our study, out of 513 specimens, ZN method showed AFB in only 3.7 percent, while culture method showed AFB growth in 10.5 percent. Another study, demonstrated 15.02 percent acid-fast bacilli by $\mathrm{ZN}$ staining method among 619 clinical specimens, while culture growth was 15.35 percent $^{15}$. In this study, culture results were used as the gold standard for assessment. Among 19 specimens which accounted for AFB positive by ZN method, only 12 specimens showed positive culture, similarly among FM staining positive specimens $(\mathrm{N}=28)$ only 11 specimens were culture positive. Thus, 7 from ZN staining and 17 from FM staining positive cases showed no growth in culture. The results from this study reemphasize the decreased sensitivity and specificity of these techniques for diagnosis of EPTB.

The maximum numbers of isolates in this study were isolated from Lymph node aspiration. The findings are concordant with similar studies in other countries ${ }^{4,12,16}$.

Forty eight of the 54 isolates were identified as $M$. tuberculosis, 4 as M. bovis, and 2 as M. avium/ intracellulare. Other studies from different part of the world ${ }^{12,17,18}$ have reported $M$. tuberculosis human type as the commonest isolates from patients with lymphadenitis. The higher incidence of $M$. tuberculosis in these studies is explained by the fact that $M$. tuberculosis complex is the most frequent cause of tuberculosis in regions like Nepal where it is rampant.

In this study, overall 62.9 percent strains showed resistance to one or more drugs. Resistance to Streptomycin was highest followed by Isoniazid ,Ethambutol and Rifampicin . The frequency of singledrug resistance was 24 percent; similar studies from Dhaka showed 29.7 percent resistance to at least one $\operatorname{drug}^{19}$.

Prevalence of MDR tuberculosis has been shown to vary widely over different regions, with the highest rates being found in Nepal (48\%), Gujrat (India) (34\%), New York City (USA) (30\%), Bolivia (15\%) and South Korea $(15 \%)^{20}$. However, HMG Nepal has documented a variable range of MDR from 0 to $18.1 \%$ on the basis of several studies ${ }^{21}$.

\section{Conclusion}

M. tuberculosis is the commonest isolate in EPTB.

These patients may thus constitute a reservoir the bacilli and ensure this survival and transmission, posing a problem for effective control.
Hence, a large longitudinal study with focused attention on EPTB is necessary to appropriate the trends of this killer disease. Simultaneous efforts to improve laboratory methods will be a great advantage.

\section{References}

1. Murry CJ, Styblo LK, Rouillon A. Tuberculosis in the developing countries: burden, intervention and cost. Tuberc Lung Dis. 1990;65:6-24.

2. HMG Ministry of health, Nepal. Annual report of Tuberculosis control programme Nepal 2002/03. Bhaktapur Nepal: HMG Ministry of health, Nepal; 2003.

3. Maher D, Chaulet P, Spinaci S, Harries A. Treatment of tuberculosis: guidelines for national programmes. Geneva: WHO; 1997.

4. Fanning A. tuberculosis: 6. Extra-pulmonary disease. CMJA. 1999;160:1597-603.

5. Chauhan MM. Non-tuberculous mycobacteria isolated from an epidemiological survey in rural population on Banglore district. Ind J Tub. 1993;40:195-7.

6. Falkinham JO. Epidemiology of infection by non-tuberculous mycobacteria. Clin Microbiol Rev. 1996;9:177-215.

7. Germa F, FitzGerald JM. Extra-pulmonary tuberculosis: two case reports. Br Columb Med J. 2002; 44:27-9.

8. Venketaraman P, Paramasivan CN. Mycobacteriology laboaratory manual-Smear examination, culture and drug susceptibility tests. Chennai, India:Tuberculosis research center; 1999.

9. Jo Baron E, Peterson RL, Finegold SM. Mycobacteria. In: Shanahan JF, editor. Bailey and scott's diagnostic microbiology. Philadelphia: Mosby; 1990.p. 590-633.

10. Reider HL, Chonde TM, Myking H, Urbanczik R, Laszlo A, Kim SJ, et al. Surveilance of antituberculosis drug resistance. In IUALTD, editors. The public health service national tuberculosis reference laboratory and the national laboratory network: Minmum requirements, role and operation in a low-income country. France. 1998; 57-82.

11. Idigoras $\mathrm{P}$, Perez-Trallero E, Alcorta $\mathrm{M}$, Gutierrez C, Munoz-Baroja I. Rapid detection of tuberculous and nontuberculous mycobacteria by microscopic observation of growth on Middlebrook 7H11 agar. Eur J Clin Microbiol Infect Dis. 1995;14:6-10.

12. Sachdeva R, Gardre DV, Talwar V. Characterization and drug susceptibility patterns of extra-pulmonary mycobacterial isolates. Ind J Med Res. 2002;115:102-7. 
13. Maltezou HC, Spyridis P, Kaftzis DA. Extrapulmonary tuberculosis in children. Arch Dis Child. 2000;83(4):342-6.

14. World Health Organization. Report on the Tuberculosis Epidemic. Geneva, Switzerland: World Health Organization; 1997.

15. Julka RK, Deb M, Patwari AK. Tuberculosis meningitis and miliary tuberculosis in children; a clinico-bacteriological profile. Ind Journ Tuberc. 1988;45(1):19-22.

16. Ilgazli A, Boyaci H, basyigit I, Yildiz F. Extra-pulmonary tuberculosis: clinical and epidemiologic spectrum of 636 cases. Arch Med Res. 2004;35(5):435-41.

17. Gadre DV, Singh UR, Talwar V. Diagnosis of tubercular cervical lymphadenitis by FNAC, microscopy and culture. Ind J Tuberc. 1991;38:25-7.

18. Dravid MN, Joshi S, Bhardwaj RS, Khare PM. "Differential identification of Mycobacterium tuberculosis from various clinical specimens from Sassoon General Hospital, Pune.” Ind J Med Sci. 1992; 46(2):43-5.

19. Riantawan P, Punnotok J, Chaisuksuwan R, Pransujarit V. Resistance of Mycobacterium tuberculosis to antituberculosis drugs in the Central Region on Thailand, 1996. Int J Tuberc Lung Dis. 1998; 2(8):609-11.

20. Cohn DL, Bustreo F, Raviglione MC. Drug resistant tuberculosis: Review of the worldwide situation and the WHO/IUATLD Global Surveilance Project. International Union against Tuberculosis and Lung Disease. Clin Infect Dis. 1999; 24(S1): 121-30.

21. HMG Ministry of health, Nepal. Annual report of Tuberculosis control programme Nepal 2001/02. Bhaktapur Nepal: HMG Ministry of health, Nepal; 2002. 